\title{
Benzodiazepine and z-hypnotic use in Norwegian elderly, aged 65-79
}

\author{
C. Ineke Neutel ${ }^{1}$, Svetlana Skurtveit ${ }^{2,3}$ and Christian Berg ${ }^{2}$ \\ 1) Department of Epidemiology and Community Medicine, Faculty of Medicine, University of Ottawa, \\ Ottawa, Ontario, Canada \\ 2) Department of Pharmacoepidemiology, Norwegian Institute of Public Health, Oslo, Norway \\ 3) Norwegian Centre for Addiction Research, University of Oslo, Norway \\ Correspondence: Christian Berg, Department of Pharmacoepidemiology, Norwegian Institute of Public Health, P.O. Box 4404 Nydalen, NO-0403 Oslo, Norway \\ Christian.Berg@fhi.no
}

\begin{abstract}
Purpose: Benzodiazepines/z-hypnotics (BZD-Z) guidelines suggest that elderly people ought to use anxiolytic benzodiazepines (BZD) and z-hypnotics only at low dose and only for a short time, and that hypnotic BZD not should be used at all. Since the elderly aged 65-79 tend to be recently retired but still in relatively good health, they may have different needs for BZD-Z than those older or younger. Our objective is to examine BZD-Z use in this age group.

Methods: The study population consisted of Norwegians, aged 65-79, who filled prescriptions for anxiolytic BZD, hypnotic BZD and/or z-hypnotics in 2004-2009. The quantities prescribed were in daily defined doses (DDD), and $100 \mathrm{DDD} /$ year was deemed excessive.

Results: More than a quarter of the population received at least one BZD-Z prescription each year. Half of those received more than $100 \mathrm{DDD} /$ year and a quarter received over $250 \mathrm{DDD} /$ year, with these proportions increasing year by year. All three subgroups of BZD-Z showed increasing use with age and all except anxiolytic BZD showed increasing proportions of users using more than 100 DDD/year with age.

Conclusions: Substantial numbers of elderly aged 65-79 receive prescriptions for BZD-Z, more with increasing age, and greater amounts per user. Guidelines are clearly ignored. While a rigid enforcement of guidelines/rules is not the answer, allowing the status quo to continue shows lack of respect for guidelines.
\end{abstract}

\section{INTRODUCTION}

Benzodiazepines and z-hypnotics (BZD-Z) have received a great deal of attention from clinical authorities and researchers regarding proper use. ${ }^{1,2}$ This has led to clinical guidelines and recommendations which can be used to group BZD-Z use into appropriate and inappropriate use categories. The best known guidelines are the Beers criteria but others followed. ${ }^{2,3}$ Although there are some differences among them, there is a general agreement that the elderly are to be prescribed only the lowest doses of BZD-Z and only for a short period of time, and that hypnotic BZD should not be used by the elderly at all.

Research has shown that many elderly use BZD-Z contrary to recommendations. ${ }^{4-8}$ One would hope that after many warnings and repeated emphasis on limiting use, at least see some trend towards improvement in use would be seen. Our objectives then are to examine BZD-Z use in elderly people: Is BZD-Z use changing over the years, and, if so, is it in the direction of increased compliance with guidelines? Our study population will be limited to persons aged 65-79 as opposed to those younger or older. Separating this age group from those younger and those older takes into account the unique features of this age group. At the lower boundary of this age group people are starting the life style changes associated with aging, such as retirement with its changes in daily activities. At the upper boundary, people are more likely to suffer from chronic illnesses or reside in nursing homes.

\section{MeTHODS}

All prescriptions filled at any pharmacy in Norway are registered in the Norwegian Prescription Database (NorPD) which started in 2004. ${ }^{9}$ Meaningless but unique identification (id) numbers are assigned each time data are used for research in order to maintain confidentiality. Information available with each prescription record includes personal identification (id) or birth month/year, prescriber id, sex, age and the DDD (Defined Daily Doses) of the medication prescribed. DDD is a quantitative unit of measurement defined as the assumed average maintenance dose per day for the medication's main indication for adults. ${ }^{10}$ The cut-off point for excessive use for this study was put at 100 $\mathrm{DDD} /$ year which was higher than the $90 \mathrm{DDD} /$ year set by another study, ${ }^{11}$ in order to err on the side of acceptable use.

Medications were coded using the Anatomical Therapeutic Chemical (ATC) classification. ${ }^{9,10,12}$ ATC codes were used to select the BZD-Z and to divide them into three subgroups: 1) anxiolytic benzodiazepines (BZD): diazepam, oxazepam and alprazolam, ATC N05BA, and clonazepam, ATC N03AE01, 2) hypnotic/sedative BZD: nitrazepam and flunitrazepam, ATC N05CD, and 3) z-hypnotics: zopiclone and zolpi- 
Table 1. Anxiolytic/sedative/hypnotic medications used by Norwegians aged 65-79 who filled at least one prescription for BZD-Z in year specified.

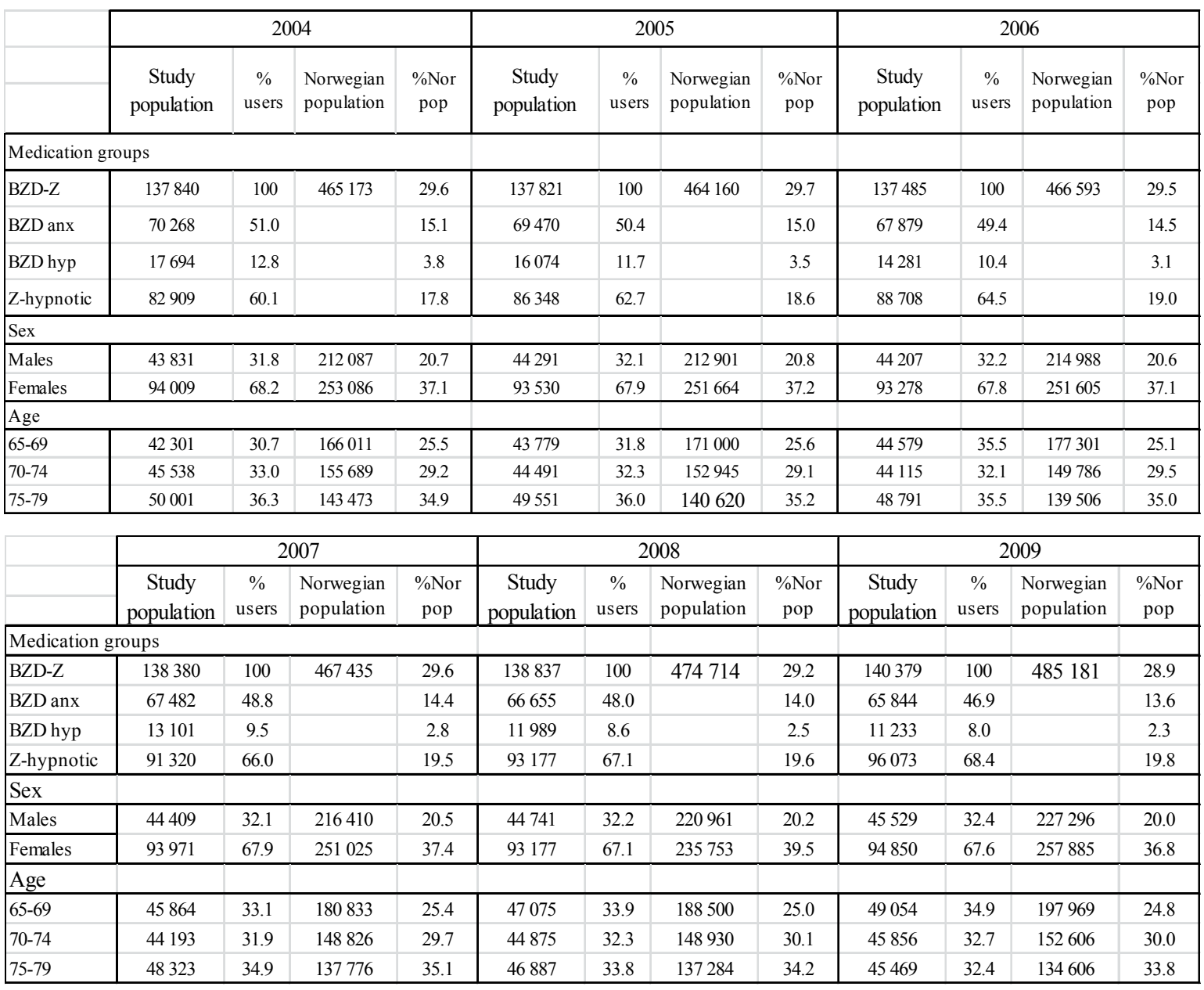

BZD-Z: Benzodiazepines and z-hypnotics

BZD anx: Anxiolytic benzodiazepines (BZD): diazepam, oxazepam and alprazolam, ATC N05BA, and clonazepam, ATC N03AE01

BZD hyp: Hypnotic/sedative benzodiazepins: nitrazepam and flunitrazepam, ATC N05CD

Z-hypnotic: Z-hypnotics: zopiclone and zolpidem, ATC N05CF

dem, ATC N05CF. The present study population was based on prescriptions filled during the years 20042009 by people aged 65 to 79 in the year specified. Excluded were persons who received medication for treatment of alcohol or opioid dependency (ATC N07BB, N07BC) at any time during the study period. The proportion of people filling prescriptions for BZD-Z in this age group changed from 29.6\% $(n=137840)$ in 2004 to $28.9 \%(n=140379)$ in 2009 (table 1). For the statistical analysis, SAS version 9.2 (SAS Institute, Cary, NC, USA) was used. Moving averages were used for all the figures. Denominator information on the Norwegian population was downloaded from Statistics Norway (http://statbank.ssb.no).

\section{RESUlTS}

Every year almost $30 \%$ of the Norwegian population filled at least one prescription for BZD-Z with most of the prescriptions being for z-hypnotics (table 1). More than twice as many women filled prescriptions for BZD-Z then did men, and more prescriptions were filled by the oldest age group than by those younger. Table 2 indicate that quantities of medication taken per user increased over this period. For example, the use over $100 \mathrm{DDD} /$ year of BZD-Z steadily increased from $52.7 \%$ in 2004 to $55.5 \%$ of users in 2009 . Similarly, the use of over $250 \mathrm{DDD} /$ year use increased from $26.2 \%$ to $29.1 \%$. Among the subgroups, hypnotic BZD showed the highest DDD/year per user followed by zhypnotics, both showing considerable increase over the years.

The figures 1-4 present the age distribution of users for the three subgroups combined, BZD-Z, (figure 1) and the three subgroups separately (figures 2-4), all stratified by year. All the $\mathrm{x}$-axes show age in single years, while the $y$-axes show percentages of the Norwegian population using the BZD-Z specified. Note that the scale of the $y$-axes differs for each graph and that the minimum is not 0 . The comparisons are to be made within each graph and these differences were made clearer by limiting the scale. The a) graph of each figure presents the percentage of the Norwegian population who filled at least one prescription in the 
Table 2. Quantities of anxiolytic/sedative/hypnotic use by Norwegians aged 65-79 who filled at least one prescription for BZD-Z in year specified.

\begin{tabular}{|c|c|c|c|c|c|c|}
\hline & 2004 & 2005 & 2006 & 2007 & 2008 & 2009 \\
\hline \multicolumn{7}{|c|}{ Percent users taking 100 DDD or more } \\
\hline BZD-Z & 52.7 & 54.2 & 54.5 & 55.0 & 55.1 & 55.5 \\
\hline BZD anx & 32.4 & 33.0 & 33.1 & 32.6 & 32.5 & 32.5 \\
\hline BZD hyp & 66.6 & 67.3 & 68.5 & 69.3 & 69.7 & 69.1 \\
\hline Z-hypnotic & 55.2 & 56.9 & 57.6 & 58.5 & 59.0 & 59.6 \\
\hline \multicolumn{7}{|c|}{ Percent users taking 250 DDD or more } \\
\hline BZD-Z & 26.2 & 27.7 & 27.9 & 28.4 & 28.8 & 29.1 \\
\hline BZD anx & 11.3 & 11.8 & 11.7 & 11.6 & 11.8 & 11.8 \\
\hline BZD hyp & 35.1 & 36.7 & 38.2 & 39.1 & 39.7 & 39.6 \\
\hline Z-hypnotic & 25.4 & 27.2 & 27.8 & 28.8 & 29.4 & 29.9 \\
\hline \multicolumn{7}{|l|}{$\begin{array}{l}\text { Mean } \\
\text { DDD }\end{array}$} \\
\hline BZD-Z & 181 & 190 & 192 & 195 & 197 & 198 \\
\hline BZD anx & 103 & 105 & 102 & 105 & 105 & 105 \\
\hline BZD hyp & 215 & 223 & 228 & 234 & 239 & 239 \\
\hline Z-hypnotic & 170 & 177 & 180 & 184 & 187 & 189 \\
\hline \multicolumn{7}{|c|}{ Median DDD } \\
\hline BZD-Z & 100 & 100 & 100 & 100 & 100 & 105 \\
\hline BZD anx & 50 & 50 & 50 & 50 & 50 & 50 \\
\hline BZD hyp & 150 & 150 & 150 & 180 & 180 & 180 \\
\hline Z-hypnotic & 100 & 113 & 120 & 120 & 120 & 120 \\
\hline
\end{tabular}

BZD-Z: Benzodiazepines and z-hypnotics

BZD anx: Anxiolytic benzodiazepines (BZD): diazepam, oxazepam and alprazolam, ATC N05BA, and clonazepam, ATC N03AE01

BZD hyp: Hypnotic/sedative benzodiazepins: nitrazepam and flunitrazepam, ATC N05CD

Z-hypnotic: Z-hypnotics: zopiclone and zolpidem, ATC N05CF

calendar year by year of study period and single years of age, while the b) graph shows the proportion of users taking $100 \mathrm{DDD} /$ year or more, also by age and stratified for years of study period.

Figure 1 presents the data for the use of the combined BZD-Z group and show increased use with age. Not only did the number of users increase with age, the proportion of users with over $100 \mathrm{DDD} /$ year also increased by age. Anxiolytic BZD also showed an increase in use by year of age (figure 2a). Anxiolytic BZD were the only one of the three BZD-Z subgroups who did not show increasing proportions of users with more than $100 \mathrm{DDD} /$ year with increasing age (figure 2b). Hypnotic BZD users were the only BZD-Z subgroup showing decreasing proportion of users over the years as shown by the 2004 curve being the highest curve (figure $3 \mathrm{a}$ ). However, the quantity of hypnotic BZD per user continued to increase by small amounts (figure $3 b$ ). Both percentage of users of z-hypnotics and quantities used by users increased per single year of age (figure 4).

\section{Discussion}

Each year more than a quarter of the Norwegians between the ages of 65-79 filled at least one prescription for BZD-Z. At least half of these users accumulated quantities over $100 \mathrm{DDD} /$ year which is excessive according to guidelines. At least a quarter of the users exceeded $250 \mathrm{DDD} /$ year which is an amount which would be sufficient for daily use according to the recommendations for the age group. Of the three BZD$\mathrm{Z}$ subgroups, the $\mathrm{z}$-hypnotics had the most users and continued increasing in use at a steady rate. Anxiolytic BZD and hypnotic BZD were used by smaller proportions of the population. Anxiolytic BZD showed a decrease in use form $15.1 \%$ in 2004 to $13.6 \%$ in 2009 , while use of hypnotic BZD decreased from 3.8\% in 2004 to $2.3 \%$ in 2009 . Z-hypnotics, however, showed an increasing proportion of users from $17.8 \%$ in 2004 to $19.8 \%$ in 2009 . 140379 people in Norway, aged 6579 , filled one or more prescriptions for BZD-Z in 2009 and 77859 of these used over 100 DDD over the year 2009. These are substantial numbers in a country of five million people. The development is in line with recommendations that especially long action hypnotic BZD should be avoided in these age groups. ${ }^{3}$

Strengths of the data include the availability of accurate data on prescription use on the complete population. There is some concern that the data for year 2004 is not as complete as subsequent years since it was the first year of the NorPD registry. ${ }^{9,12}$ There is no evidence in the tables and graphs that the 2004 data differs from the other years. In fact, the data for the year 2004 fits in very well with the trends, both when the 2004 data are the lowest, as in most graphs, or when they are the highest as for hypnotic BZD (figure 3a). A potential limitation of the data is that the lack of actual daily dose prescribed and/or consumed is unknown. What was available was the total amount DDD/year and while not ideal, conclusions could be drawn. For example, the proportion of people receiving over $100 \mathrm{DDD} /$ year provides a useful estimate of excess use.

Patterns of BZD-Z use can be seen in three ways: 1) BZD-Z use in the population, 2) quantities of BZD-Z received per user, 3) age-specific BZD-Z use.

1) BZD-Z use in the population. BZD-Z were used by almost $30 \%$ of the Norwegian population of this age group. While overall proportions of BZD-Z use did not change by much over the six years of the study, the proportions of the subgroups were changing. As a proportion of BZD-Z users, both hypnotic and anxiolytic BZD use were slowly declining while z-hypnotics use, already the highest, continued to increase. As explanation of this trend, Hausken et al. suggest that z-hypnotic are chosen over BZD because z-hypnotics are shorteracting than the BZD available in Norway. ${ }^{13}$

2) Quantities of BZD-Z used. More than half of users received over $100 \mathrm{DDD} /$ year and this proportion was increasing especially for z-hypnotics. According to 
a. Proportion of Norwegian population using BZD-Z
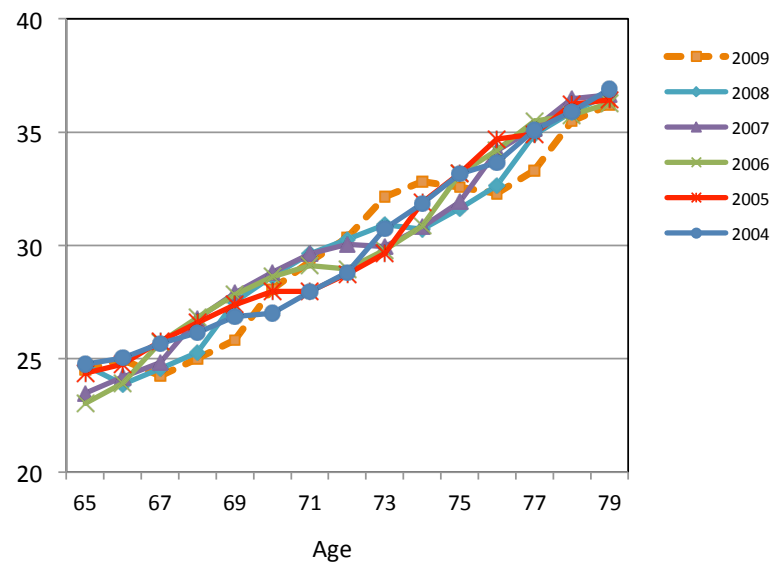

b. Proportion of users with 100 DDD or more in year
specified

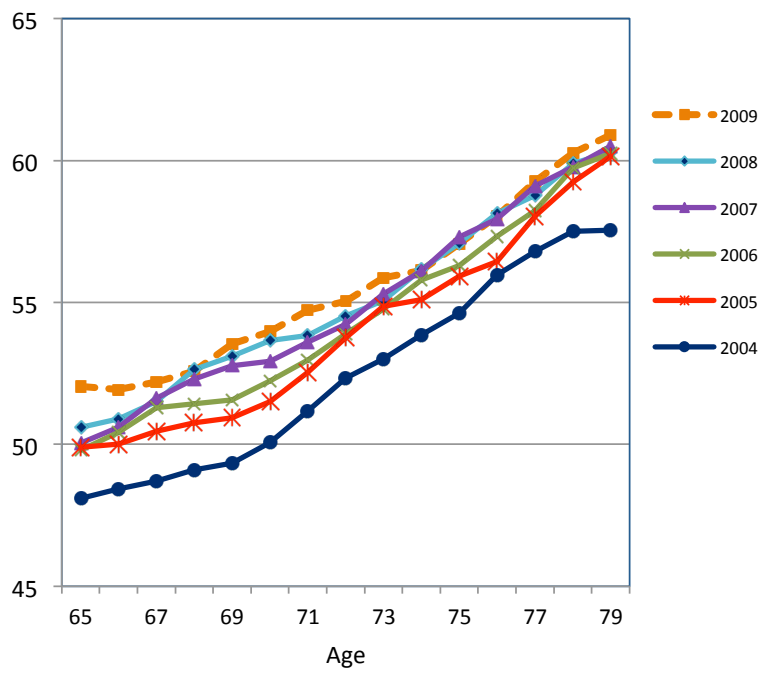

Figure 1. BZD-Z use by age for Norwegians aged 65-79 who used at least one prescription for BZD-Z in year specified.

guidelines only low doses are recommended for the elderly, which for z-hypnotics and hypnotic BZD is 0.75 DDD per day, somewhat higher for anxiolytic BZD. ${ }^{3,14}$ The 100 DDD/year cut-off would allow a daily low dose for well over four months of use which is well over the suggested limit of no more than one month at a time. As half of users (or $15 \%$ of the population) exceeding the $100 \mathrm{DDD} /$ year and $25 \%$ of users (or $8 \%$ of the population) exceeding $250 \mathrm{DDD} / \mathrm{year}$, it is clear that there is a widespread lack of adherence to guidelines. These finding confirm other studies in Norway and elsewhere, some of which are a decade old. $^{15-18}$ We may conclude that there has been little progress in this area.

3) Age-specific BZD-Z use. Another interesting finding is the pattern of increasing use with increasing age even within the narrow age range of this population. Note that the measure of 100 DDD/year includes both daily dose and length of time of use. Increasing use with age is consistent with the increasing chronic disease and other discomforts associated with age. The concern
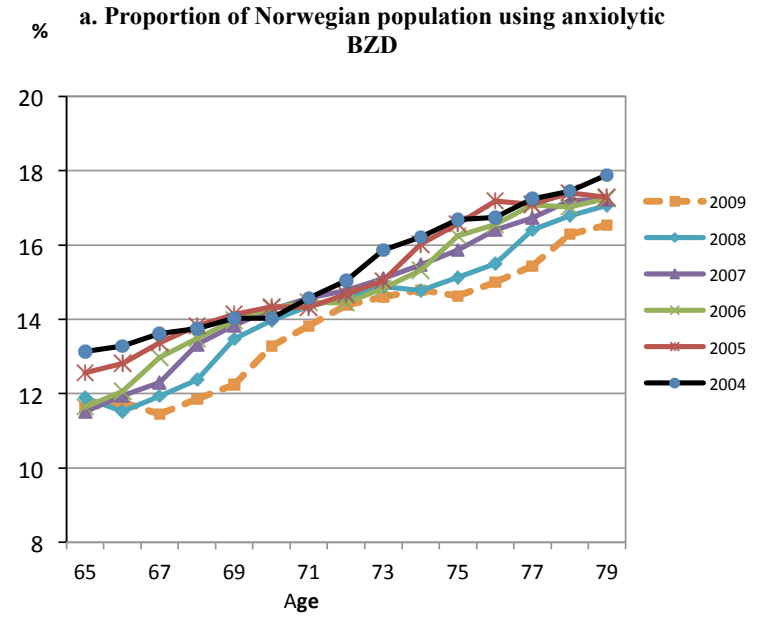

\% b. Proportion of users with 100 DDD or more in year specified

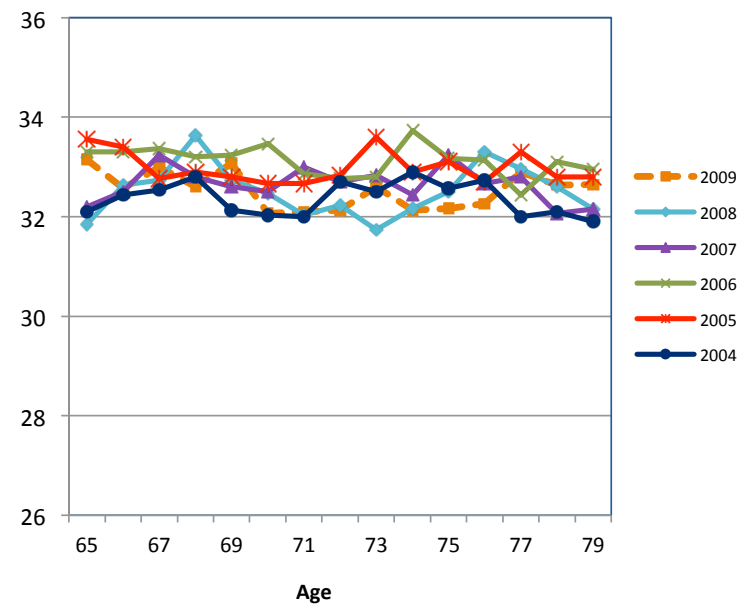

Figure 2. Anxiolytic BZD use by age for Norwegians aged 65-79 who received at least one prescription of anxiolytic BZD in year specified.

is that the elderly with the uncomfortable conditions warranting sleeping pill use are also the ones who use more other medications. The resulting polypharmacy may lead to side effects, drug interaction, etc.

While guidelines are not relevant to the substantial proportions of the population using BZD-Z, nor to the growing use with increasing age, the guidelines do cover length of time and quantities used. The increasing quantities of BZD-Z per user are definitely not according to guidelines, nor are annual trends in the direction of increasing number of DDDs/per year. The only bright light is the decreasing use of hypnotic BZD, however, minimal. Studies in other countries have also shown the improper, i.e., the beyond guideline, use BZD-Z by elderly. ${ }^{4-8}$ Our results have shown that in Norway for most years more than half of users used well over the maximum amounts suggested by guidelines. In numbers this means that more than 70000 Norwegians are taking excess BZD-Z annually just for this age group. 

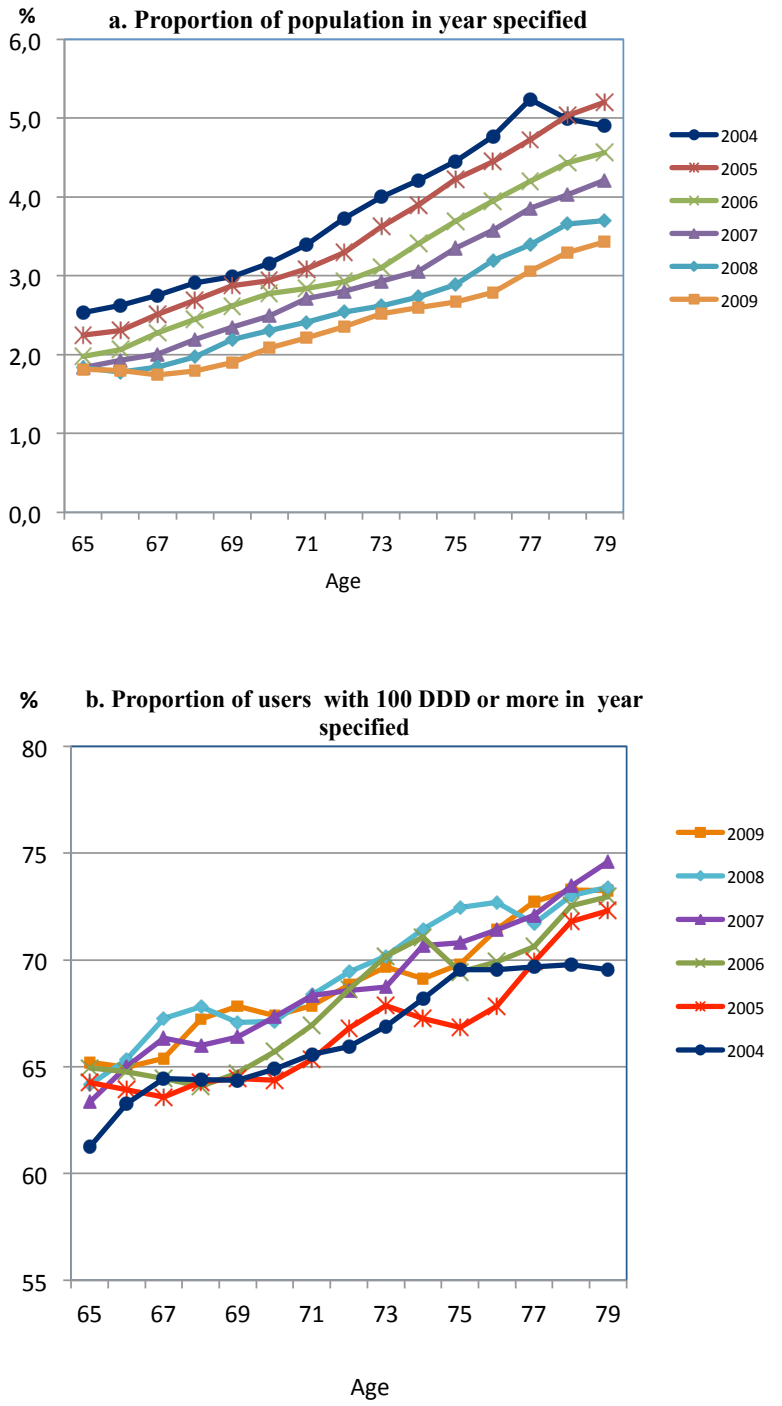

Figure 3. BZD-hypnotic use by age for Norwegians aged 65-79 with at least one prescription for hypnotic BZD in year specified.

The policy makers and prescribers have a number of options in response to the increasing use of BZD-Z beyond generally accepted guidelines. The first option is to continue the status quo and ignore guidelines, and continue to publish studies showing the large proportion of people ignoring guidelines. This is the default option and will have the result that developing further guidelines will be pointless. The second option is to strictly enforce the guidelines no matter what. This would take a commitment and buy-in by physicians, as well as somewhat of a hard heart towards their elderly patients. Difficult decisions would need to be made about which patients have legitimate needs and which do not. Past research has shown that rigid enforcement may result in persons with legitimate needs being the ones most likely to go without. ${ }^{19-21}$ Reaching these goals by regulatory or other interventions in BZD-Z in the elderly shows variable results. ${ }^{22-25}$ However, there are substantial differences between the health systems in the countries. In Norway the general practitioner scheme which defines a regular physician for each patient and hence continuity in the treatment, provides a
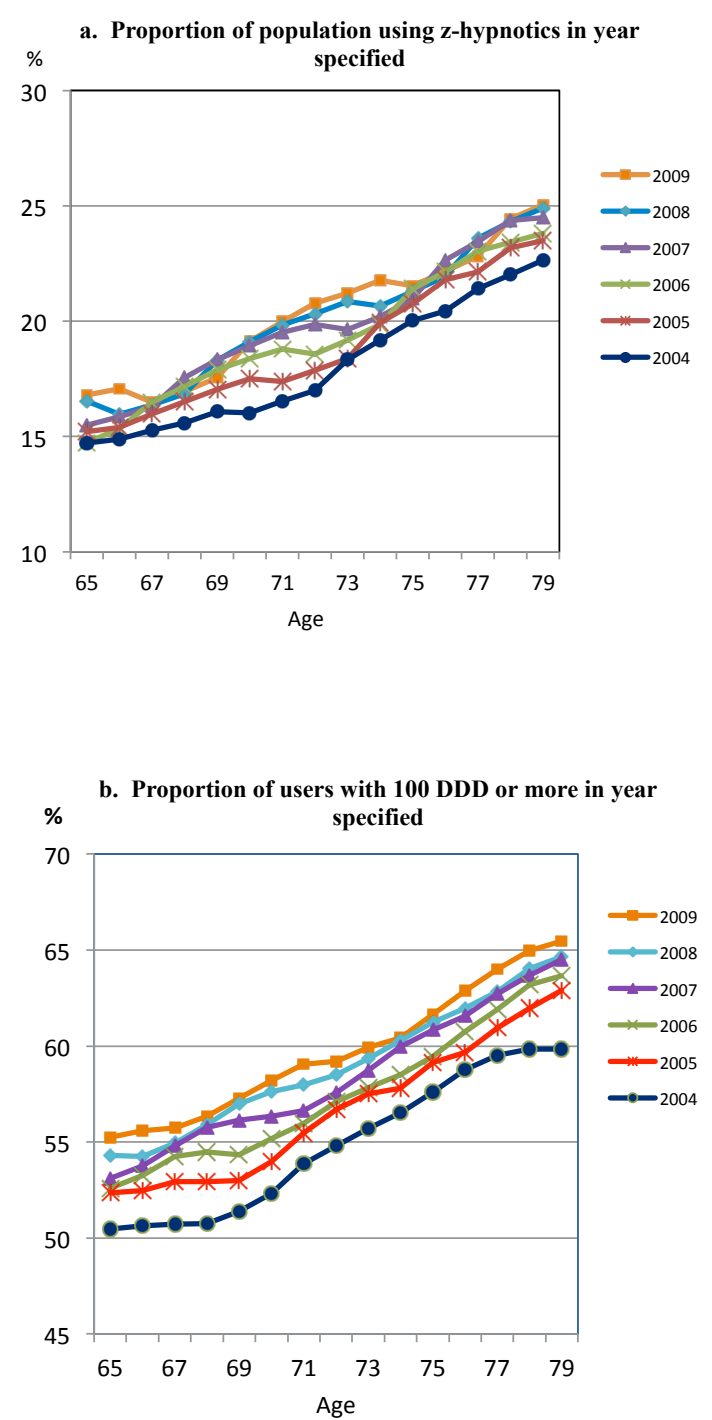

Figure 4. Z-hypnotic use by age for Norwegians aged 65-79 with at least one prescription for Z-hypnotic in the year specified.

good possibility to a close follow up of the BZD-Z users. $^{25,26}$

A third option is to re-evaluate the guidelines and the purpose of guidelines. Studies have shown that, on the whole, BZD-Z are effective and remarkably safe drugs. ${ }^{26,27}$ Most will also agree that many elderly suffer from insomnia or from discomforts which make sleep difficult. There are, however, also people who take their daily BZD-Z out of habit and have done so for years. While these are the ones who should reduce their use, they are probably also the most difficult to wean from BZD-Z use, whether because of fear of the rebound effect or sheer habit. In any case, realistic and enforceable guidelines are needed, otherwise the concept of guidelines becomes a laughing stock and ignored by all. Continuing to reiterate guidelines that are ignored by as many as $25 \%$ of the population and their physicians is counterproductive and must not be allowed to continue, one way or another. An objective and informed analysis of the situation is needed to produce a creative solution that is the most beneficial to patients, physicians and the health care system. 


\section{REFERENCES}

1. Ashton H. Guidelines for the rational use of benzodiazepines. When and what to use. Drugs 1994; 48: 25-40.

2. Beers MH. Explicit criteria for determining potentially inappropriate medication use by the elderly. An update. Arch Intern Med 1997; 157: 1531-6.

3. Rognstad S, Brekke M, Fetveit A, Spigset O, Wyller TB, Straand J. The Norwegian General Practice (NORGEP) criteria for assessing potentially inappropriate prescriptions to elderly patients. A modified Delphi study. Scand J Prim Health Care 2009; 27: 153-9.

4. Johnell K, Fastbom J. The use of benzodiazpines and related drugs amongst older people in Sweden: associated factors and concomitant use of other psychotropics. Int J Geriatr Psychiatry 2009; 24: 731-8.

5. Gallagher P, Barry P, O'Mahony D. Inappropriate prescribing in the elderly. J Clin Pharm Ther 2007; 32: 11321.

6. Bregnhoj L, Thirstrup S, Kristensen MB, Bjerrum L, Sonne J. Prevalence of inappropriate prescribing in primary care. Pharm World Sci 2007; 29: 109-15.

7. Gorgels WJ, Oude Voshaar RC, Mol AJ, Breteler MH, van de Lisdonk EH, Zitman FG. [Long-term use of benzodiazepines]. Ned Tijdschr Geneeskd 2001; 145: 1342-6.

8. Lagnaoui R, Depont F, Fourrier A, et al. Patterns and correlates of benzodiazepine use in the French general population. Eur J Clin Pharmacol 2004; 60: 523-9.

9. Furu K, Wettermark B, Andersen M, Martikainen JE, Almarsdottir AB, Sorensen HT. The Nordic countries as a cohort for pharmacoepidemiological research. Basic Clin Pharmacol Toxicol 2010; 106: 86-94.

10. WHO Collaborating Centre for Drugs Statistics Methodology. Oslo, Norway, available from: www.Whocc.no (last accessed 02/01/2012); 2010.

11. Nelissen-Vrancken (ed.). Werkgroep Prescriptiekoppeling: Werken met cijfers in het FTO. Utrecht, 2003.

12. Furu K. Drug utilisation in a public health perspective: Establishing a national prescription register in Norway. Norsk Epidemiologi 2001; 11: 55-60.

13. Hausken AM, Furu K, Skurtveit S, Engeland A, Bramness JG. Starting insomnia treatment: the use of benzodiazepines versus z-hypnotics. A prescription database study of predictors. Eur J Clin Pharmacol 2009; 65: 295-301.

14. Rønning M, Berg C, Furu K, et al. The Norwegian Prescription Database, 2004-2009. Oslo: The Norwegian Institute of Public Health, 2010.

15. Berg C, Sakshaug S, Handal M, Skurtveit S. Z-hypnotics - The dominating hypnotics in Norway. Norsk Farmaceutisk Tidsskrift 2011; 4: 20-3.

16. Bartlett G, Abrahamowicz M, Tamblyn R, Grad R, Capek R, du Berger R. Longitudinal patterns of new benzodiazepine use in the elderly. Pharmacoepidemiol Drug Saf 2004; 13: 669-82.

17. Straand J, Rokstad KS. Elderly patients in general practice: diagnoses, drugs and inappropriate prescriptions. A report from the More \& Romsdal Prescription Study. Fam Pract 1999; 16: 380-8.

18. Straand J, Rokstad K. General practitioners' prescribing patterns of benzodiazepine hypnotics: are elderly patients at particular risk for overprescribing? A report from the More \& Romsdal Prescription Study. Scand J Prim Health Care 1997; 15: 16-21.

19. Ross-Degnan D, Simoni-Wastila L, Brown JS, et al. A controlled study of the effects of state surveillance on indicators of problematic and non-problematic benzodiazepine use in a Medicaid population. Int J Psychiatry Med 2004; 34: 103-23.

20. Simoni-Wastila L, Ross-Degnan D, Mah C, et al. A retrospective data analysis of the impact of the New York triplicate prescription program on benzodiazepine use in medicaid patients with chronic psychiatric and neurologic disorders. Clin Ther 2004; 26: 322-36.

21. Wagner AK, Soumerai SB, Zhang F, et al. Effects of state surveillance on new post-hospitalization benzodiazepine use. Int J Qual Health Care 2003; 15: 423-31.

22. Kaur S, Mitchell G, Vitetta L, Roberts MS. Interventions that can reduce inappropriate prescribing in the elderly: a systematic review. Drugs Aging 2009; 26: 1013-28.

23. Jorgensen VR. Benzodiazepine reduction in general practice. Are the elderly neglected? J Affect Disord 2012; 136: 1216-21.

24. Mouland G. [A letter to benzodiazepine users - an efficient way to reduce the prescription]. Tidsskr Nor Lægeforen 1997; 117: 3097-100.

25. Straand J, Fetveit A, Rognstad S, Gjelstad S, Brekke M, Dalen I. A cluster-randomized educational intervention to reduce inappropriate prescription patterns for elderly patients in general practice - The Prescription Peer Academic Detailing (Rx-PAD) study [NCT00281450]. BMC Health Serv Res 2006; 6: 72.

26. Ancoli-Israel S, Richardson GS, Mangano RM, Jenkins L, Hall P, Jones WS. Long-term use of sedative hypnotics in older patients with insomnia. Sleep Med 2005; 6: 107-13.

27. van Hulten R, Teeuw B, Bakker A, Leufkens HG. Initial benzodiazepine use and improved health-related quality of life. Pharm World Sci 2005; 27: 41-6. 